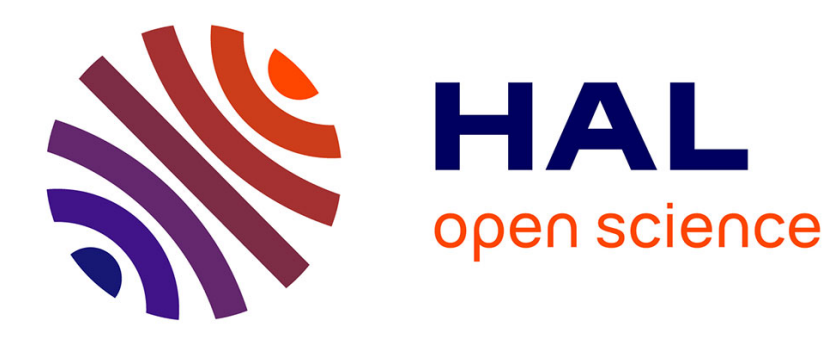

\title{
Conventions et Acadepreneuriat au cœur de la gouvernance universitaire
}

Thierry Levy-Tadjine

\section{To cite this version:}

Thierry Levy-Tadjine. Conventions et Acadepreneuriat au cœur de la gouvernance universitaire. La Gouvernance Universitaire, Feb 2009, Jounieh, Liban. hal-00418522

\section{HAL Id: hal-00418522 \\ https://hal.science/hal-00418522}

Submitted on 19 Sep 2009

HAL is a multi-disciplinary open access archive for the deposit and dissemination of scientific research documents, whether they are published or not. The documents may come from teaching and research institutions in France or abroad, or from public or private research centers.
L'archive ouverte pluridisciplinaire HAL, est destinée au dépôt et à la diffusion de documents scientifiques de niveau recherche, publiés ou non, émanant des établissements d'enseignement et de recherche français ou étrangers, des laboratoires publics ou privés. 


\section{Conventions et Acadepreneuriat au cour de la gouvernance universitaire}

\section{Thierry LEVY-TADJINE}

Professeur- Associé à l'Université St Esprit de Kaslik (USEK, Liban)

Directeur du CIRAME (Centre de Recherches en Gestion de l’USEK)

Membre du Laboratoire ICI - Université de Bretagne Occidentale, thierrylevy@usek.edu.lb

Résumé : L'ambition de cette contribution exploratoire est de montrer que l'approfondissement du concept d'acadépreneuriat et le recours à la théorie des conventions permettent de porter un regard nouveau et pragmatique sur la gouvernance universitaire surtout dans le contexte moderne marqué par une démultiplication des attentes de la société à l'égard des institutions éducatives de l'enseignement supérieur.

Mots clefs: Gouvernance Universitaire ; Parties prenantes ; Acadépreneuriat ; Conventions.

Abstract : Underlining two paradoxes for academic governance in the modern context, this text suggests that mobilizing Acadépreneurship in practice and French Conventions' Theory is useful for defining the good but diversified practices for universities' government.

Key-Words : Academic Governance ; Shareholders ; Acadepreneurship ; Conventions. 


\title{
Conventions et Acadepreneuriat au cour de la gouvernance universitaire
}

\author{
Thierry LEVY-TADJINE ${ }^{1}$ \\ Professeur- Associé à l'Université St Esprit de Kaslik (USEK, Liban) \\ Directeur du CIRAME (Centre de Recherches en Gestion de l'USEK) \\ Membre du Laboratoire ICI - Université de Bretagne Occidentale, \\ thierrylevy@usek.edu.lb
}

\section{Introduction: Une problématique renouvelée de la Gouvernance Universitaire}

Notre réflexion part d'un double constat. En premier lieu, les missions assignés à l'Université par la société s'accroissent. En second lieu et corrélativement, le nombre de parties prenantes de l'Université augmente. Ces deux facteurs avérés par la littérature comme par l'expérience de l'auteur dans plusieurs institutions universitaires en France, au Liban et en Tunisie, posent à l'Université, un problème de gouvernance.

Selon DRUCKER (1957), «la gouvernance consiste à mettre au point des règles qui guident et limitent la conduite de ceux qui agissent au nom de l'entreprise ».

Par la suite, le concept de gouvernance a été alimenté par la théorie principal-agent et LEVET (1999) la définit comme modalité du «contrôle de propriété exercé sur les entreprises par les actionnaires ».

Par extension de ces applications aux relations entre actionnaires et dirigeants, la gouvernance désigne l'ensemble des dispositions qui permettent à des individus délégant un pouvoir à d'autres agents de vérifier que ces derniers agissent bien dans leurs intérêts. Ainsi, il est devenu légitime d'appliquer le concept de gouvernance au système universitaire. L'enjeu est alors tantôt de pouvoir évaluer comment les institutions servent bien les intérêts de leurs clients (étudiants ; entreprises...) notamment par la production de qualifications valorisées par le marché du travail ${ }^{2}$, tantôt de mesurer comment les dirigeants des universités respectent les intérêts du contribuable lorsqu'il s'agit d'établissements publics ou des actionnaires ou organismes de tutelle dans les autres cas. Il peut aussi s'agir d'évaluer comment les enseignants assument les missions que l'université reçoit de la société civile ou de ses organismes de tutelle. L'enjeu de l'identification de ces missions est alors au cœur de notre appréhension de la gouvernance universitaire moderne, c'est pourquoi, nous y consacrerons la première section de cette contribution. Nous essaierons de montrer, en particulier, que la démultiplication des missions assignées aux universitaires n’est pas sans conséquences paradoxales sur les mécanismes de gouvernance des universités. Dans un premier temps, cela génère une diversité des modèles de gouvernance que nous nous efforcerons

\footnotetext{
${ }^{1}$ La version définitive de ce texte a bénéficiée des commentaires stimulants et des suggestions pertinentes de Jean-Yves LEROUX que nous tenons à remercier.

${ }^{2}$ Cf la contribution de J.F. VERNE («Diplômes et marché du travail : une faille dans le système de gouvernance ») à ce colloque
} 
d'appréhender dans la section 2 en recourant à la théorie des conventions. Le nouveau contexte interpelle aussi les personnels universitaires sur la conception de leur métier en remettant en cause la notion de profession universitaire et pour dépasser la tension qui en résulte, nous proposerons d'encourager les pratiques acadépreneuriales à l'université (section 3).

\section{L’Accumulation des missions de l'Université et la naissance d'un paradoxe de gouvernance.}

Comme le note LOSEGO (2004), « ce qui est commun a la plupart des universités du monde industrialisé, c'est l'accumulation des missions (qui leur sont confiées) depuis trois décennies. Aux fonctions traditionnelles d'enseignement et de recherche, se sont adjointes de plus en plus de taches administratives " qui, selon diverses enquêtes, mobiliseraient près de $40 \%$ du temps de travail des universitaires (LOSEGO, 2004).

Certains, dans le cadre de rapports, ont tenté de lister ces nouvelles missions de l'université. Ainsi aux activités de recherche et d'enseignement, ESPERET (2001) en ajoute neuf :

-l'établissement de relations internationales (échanges ; diplômes internationaux),

-la contribution au développement local (liens avec les entreprises et les collectivités locales),

-la valorisation de la recherche (par la mise en place d'incubateurs notamment),

-l'expertise,

-l'évaluation des formations et de la recherche,

-la professionnalisation des formations notamment par l'assistance aux étudiants dans leur recherche de stage,

-la formation continue,

-l’ingénierie de formation,

-et, enfin, plus étonnamment, l'action culturelle (manifestations culturelles, patrimoine, etc).

Parallèlement, d'autres auteurs, héritiers de la philosophie sociale et pragmatique de John $\mathrm{DEWEY}^{3}$, considèrent que l'Université et ses membres ont également pour mission, d'éclairer le débat politique. Pour le célèbre Professeur d'Entrepreneuriat de l'ESSEC, BOUCHIKI (2004), la recherche a systématiquement trois audiences (académique; pratique et politique). Si l'orientation académique de la recherche permet de distinguer l'université des collèges universitaires, l'enseignement et la recherche s'enrichissant réciproquement par un mécanisme de fertilisation croisée, l'orientation politique suggère que l'université doit autant etre ouverte au monde de l'entreprise (orientation pratique dans les propos de BOUCHIKI) qu’aux décideurs macroéconomiques et institutionnels.

Cette liste sous-tend une liste élargie de parties prenantes. Si autrefois, comme le rappellent BEN-HAMOUDA et PATUREL (2009) à ce colloque, on pouvait limiter les

\footnotetext{
${ }^{3}$ Comme le note COULON (2004; 12), « selon le pragmatisme, le philosophe est impliqué dans la vie de sa cité, s'intéresse à son environnement, à l'action sociale qui a pour but le changement social. (...) Le pragmatisme est, en effet, une philosophie de l'action qu'on pourrait également appeler une philosophie de l'action sociale».
} 
parties prenantes au «triangle académique » (CLARK, 1983), mettant l’Université au cœur des relations entre trois pôles que sont la régulation du gouvernement, l'oligarchie académique et le marché, les nouvelles typologies des parties prenantes universitaires sont beaucoup plus riches. Comme le rapportent BEN-HAMOUDA et PATUREL (op. cit.), KOTLER \& FOX (1995) retiennent seize parties prenantes sur lesquelles nous reviendrons. Car l'élargissement des missions questionne aussi le métier de l'universitaire et la manière dont il va se positionner vis-à-vis de sa hiérarchie et des parties prenantes.

La liste d'ESPERET (2001) suggère une grande diversité de compétences et on se doute qu'un même individu ne puisse réunir par exemple, des compétences interculturelles favorables au développement d'échanges internationaux, une orientation créative pour l'ingénierie de formation, des compétences andragogiques ${ }^{4}$ pour assurer les formations continues, des qualités d'animation d'un incubateur en sus de ses compétences supposées dans le domaine pédagogique et scientifique. Il n'existe alors plus de profil type de l'enseignant-chercheur universitaire et suivant LOSEGO (2004), « cette accumulation et cette complexification des taches devraient s'accompagner d'une division technique du travail ». De notre point de vue, en constatant avec cet auteur que ce n'est pas le cas au niveau des personnels universitaires qui "restent attachés à leur institution en général et sont censés participer à toutes ces missions" (op. cit.), il en résulte une division territoriales des spécialisations suivant les universités, certaines s'orientant davantage vers certaines missions que d'autres. Il ne saurait, dans ces conditions, exister un modèle unique de gouvernance. Sur le terrain, on constate par exemple que certaines institutions misent davantage sur la professionnalisation et sur la formation continue que d'autres (les IAE en France, les ISG en Tunisie par exemple). De ce fait, dans la seconde section, nous serons conduit à mobiliser la théorie des conventions pour proposer une grammaire des modes de gouvernance universitaire qui rende compte de la diversité des approches que génère la démultiplication des missions.

Cependant, l'examen des missions reconnues aux universitaires (leurs deux missions initiales et les neuf nouvellement identifiées) conduit aussi à un paradoxe sur la définition du métier même de ces derniers.

Les nouvelles missions se prêtent davantage que les missions traditionnelles à l'évaluation de la productivité de leur responsable. On peut ainsi aisément évaluer la mission internationale d'une université au nombre d'étudiants étrangers qu'elle accueille, au nombre de co-diplomations qu'elle a mis en place, etc. On peut aussi évaluer un service de formation continue par le nombre de sessions organisées en entreprises. Malgré l'émergence de classements internationaux (comme celui très contestable de Shanghai qui classe les universités du monde entier selon une approche multicritères ou comme les différents classements des revues scientifiques établis par le CNRS et par diverses écoles pour évaluer la productivité des chercheurs), d'organismes d'évaluation (l'AERES en France), de référentiels (AACSB, EQUIS...) visant à instaurer des standards internationaux, la mesure de l'efficacité du système universitaire dans

\footnotetext{
${ }^{4}$ Cf BELET (1991). Pour cet auteur, l'andragogie est entendue comme «l'art d'enseigner à des adultes par référence à la pédagogie qui est l'art d'enseigner a des enfants ou adolescents ».
} 
l'enseignement comme dans la recherche, restent délicate ${ }^{5}$. Ces deux missions ne se réduisent pas à des critères productivistes qui peuvent, dans certains cas, leur nuire. Le système du « publish or perish » pousse à l'extrême, peut conduire ainsi à une limitation de la créativité scientifique quand pour être publié dans une revue A, il faut rentrer dans le mainstream et ne pas trop s'en écarter. L'intérêt des chercheurs de Stanford pour les philosophes français de Polytechnique, plus libres qu'eux, est une illustration incontestable de ce point.

Or, cette opposition entre deux types d'activités, celles qui se prêtent aisement à l'évaluation en termes de productivité et les autres, plus immatérielles, recouvre la distinction sociologique entre «la profession » et « le travail» (STROOBANTS, 1993). Comme le note LOSEGO (2004) qui pointait cette tension et dont nous nous inspirons, « il n’y a pas de travail sans mesure de la productivité ».

Comme l'illustre FREIDSON (1984) à propos de la profession médicale, la profession est censée etre complexe, ésotérique et l'efficacité et la compétence de ceux qui l'exercent ne peuvent etre appréciées que sur le plan moral et social, par des pairs avec un regard d'expertise. CLARK (1987) montre que l'université traditionnelle relève de ce système qui entre aujourd'hui en tension avec la tendance à la standardisation des taches universitaires et avec la volonté collective de faire de l'universitaire, un travailleur comme les autres, évalué sur sa productivité et soumis à des horaires de travail fixes (au risque alors qu'il exerce sa profession avec moins d'enthousiasme et de créativité). Pour nous, cette tension manifeste l'un des paradoxes de la gouvernance universitaire contemporaine et l'Acadépreneuriat (Section 3) pourra etre vu comme une option pour le dépasser.

Cependant, du fait de l'élargissement du champ de ses missions, l’Université se trouve au cœur d'un réseau élargi de parties prenantes comme nous le rapportions plus haut. La focalisation sur telle ou telle d'entre elles induira un mode de gouvernance spécifique. Nous proposons dans la section 2 , de décrypter les différents modèles de gouvernance universitaire à la lumière des Economies de la Grandeur de BOLTANSKI et THEVENOT $(1987,1991)$ et de la théorie des conventions que GOMEZ (1996) appliquait déjà à la problématique de la gouvernance. Pour ce faire, nous nous appuierons sur le modèle de KOTLER \& FOX (1995).

\section{Les conventions d'effort et la multiplicité des modèles de gouvernance universitaire.}

Soumis au regard critique et exigeante d'un ensemble de parties prenantes, les universités sont, en effet en prise avec des registres d'évaluation différents. En reprenant la typologie de KOTLER \& FOX qui identifient seize parties prenantes pour les établissements d'enseignement supérieur (les étudiants; les futures étudiants ; les parents ; les enseignants ; le personnel administratif ; le gouvernement ; les collectivités locales ; les établissements concurrents; les mass medias; les organismes d'accréditation; les

\footnotetext{
${ }^{5}$ Comme nous le suggérait le rapporteur sur notre texte, bien qu'elle soit délicate, l'évaluation du système universitaire comme de ses membres, repose pourtant le plus souvent sur l'appréciation quantitative des recherches conduites. C'est, en effet, principalemenrt sur la base des publications scientifiques que l'AERES (Agence d'Evaluation de la recherche et de l'Enseignement Supérieur) en France, classe les établissements d'enseignement supérieur et que le Conseil National des Universités statue sur les carrières des universitaires.
} 
fournisseurs d'équipement ; le monde professionnel ; etc ), il est tautologique d'affirmer que chacun de ces acteurs ne manifestera pas les mêmes attentes envers le système universitaire et qu'il n'évaluera pas ce dernier sur les mêmes bases.

Pourtant, à un moment donné, pour une institution donnée, un accord s'établira sur la façon d'enseigner et de conduire des travaux de recherche qui satisfassent les parties prenantes.

C'est pour cette raison que la mobilisation de l'axiomatique de BOLTANSKI et THEVENOT est pertinente pour notre propos. En soulignant que la philosophie moderne s'est construite sur la base d'une compartimentation des espaces de justification, les auteurs montrent ensuite que l'analyse des pratiques et des discours des acteurs atteste, au contraire, de l'imbrication des différents critères de justification.

Identifiant six « cités » correspondant aux six critères de justification des actions qu'ils repèrent dans la littérature, ils affirment que la plupart des actions humaines font appel à des compromis entre ces énoncés de justification. BOLTANSKI et THEVENOT (1987, 1991) évoquent ainsi le «critère marchand ${ }^{6}$, le critère "industriel " ${ }^{7}$, le critère "domestique ${ }^{8}$, le critère « civique ${ }^{9}$, le critère du renom ${ }^{10}$ et le critère « divin» ${ }^{11}$ comme étant combinables pour juger justifier des actions.

Conceptuellement, le modèle des «économies de la grandeur " (BOLTANSKI et THEVENOT, op. cit.) autorise, dans toute situation sociale, une pluralité de jugements possibles et, dans le cas du système universitaire, permet de rendre compte de la multiplicité des évaluations possibles de la qualité par le client (étudiant) comme par les autres parties prenantes.

En suivant EYMARD-DUVERNAY (1989), on pourrait ainsi distinguer au moins trois conventions de qualifications selon que sur «le marché de l'enseignement supérieur », l'évaluation est "marchande" (centré sur le coût de la formation et le retour sur investissement de celle-ci), « industrielle » (focalisée sur le respect de certains standards techniques, sur des contenus ou sur une pédagogie particulière notamment...) ou "domestique » (l'évaluation reposant alors surtout sur la réputation de l'établissement). De même, l'offreur de service éducatif dispose de plusieurs registres pour évaluer et produire la qualité de ce qu'il propose sur le marché. GOMEZ (1994), se focalisant sur la production de la qualité, parlait de convention d'effort. Nous avons montré par ailleurs (LEVY, 2002) que les deux conventions d'effort, qu'il décrivait (la firme individualiste et la firme communautaire), peuvent être rapprochées des cites industrielle et marchande de BOLTANSKI et THEVENOT (1987) pour la première et civique et domestique pour la seconde.

Dans la convention communautaire, les managers universitaires s'efforcent de « donner du sens au travail des employés qui la composent » en mettant l'accent sur le collectif et en valorisant peu les actions individuelles. Dans certaines institutions adhérant à cette logique, on peut entendre des universitaires énoncer :

\footnotetext{
${ }^{6}$ Les actions sont légitimées sur la base de principes marchands.

${ }^{7}$ Les actions sont évaluées et légitimées à l'aune de standards attendus.

${ }^{8}$ C'est la tradition ou la réputation qui servent à guider les comportements.

${ }^{9}$ L'action est évaluée par rapport au bien commun.

${ }^{10} \mathrm{~L}$ 'action serait évaluée à partir de l'opinion.

${ }^{11}$ Dans la « cité de Dieu », l'action est fondée sur la grâce ou sur l’inspiration divine.
} 
« Je passe l'agrégation, mais je ne le fais pas pour moi... C'est pour l'institution. Donc j'aurais besoin de votre aide. Vos recherches me serviront....».

Une telle perspective, apparentée à "la cité civique" dans laquelle l'accord sur l'évaluation et sur l'action à mener provient du fait que tous les individus donnent priorité au bien commun sur leurs intérêts individuels, se distingue nettement d'univers plus individualistes. Dans la convention individualiste qui prévaut aujourd'hui dans la plupart des grandes écoles de commerce occidentales, l'initiative individuelle est encouragée et valorisée. Nombre d'écoles (HEC Montréal ; Sup de Co Montpellier, etc) attribuent ainsi des primes financières à leurs enseignants dés qu'un de leurs articles est publié, le montant du bonus variant selon la notoriété de la revue. En suivant GOMEZ (1994), ce modèle s'assimile aux entreprises (comme McDonald) qui affiche «l'employé du mois ». Cette dernière remarque permet de rappeler combien ces deux conventions sont des figures types. Le modèle mis en place localement peut prendre la forme d'un compromis. Ainsi, à titre d'illustration, il est notable qu'à ce jour, l'USEK qui accueille ce colloque est davantage située dans le registre communautaire pour la gestion des travaux de recherche non rétribués individuellement de ses membres mais tend vers le modèle individualiste en mentionnant chaque mois « l'alumnus du mois » sur son site web.

Une telle perspective comme l'approches plus gestionnaire de la théorie socioéconomique des organisations (SAVALL, ZARDET, 1991), tend aussi à souligner la dissociation entre évaluation interne et évaluation externe de la qualité et fait de celle-ci, le cour des questions de gouvernance universitaire.

Cette dissociation, croisée avec les différents registres par lesquels l'action universitaire, est évaluée, permet d’identifier plusieurs modèles de gouvernance universitaire que nous nous efforcerons d'illustrer et de discuter. Dans cette optique, le modèle de gouvernance universitaire résulte d'une interaction entre les orientations internes d'un établissement (convention d'effort) et les attentes de ses parties prenantes qui supportent des conventions de qualification. Le croisement génère ce que DEROUET (1992) qualifie de "convention d'éducation». Accessoirement, il est aisé de montrer qu'une telle orientation prend le contre-pied des théories économiques du capital humain. Le "bien" Education est plus qu'un simple capital dont la fonction de production serait connue et appréhendable. L'économie des conventions envisage l'économie de l'éducation comme un problème typique de coordination entre acteurs et s'intéressera alors aux solutions que les acteurs dans des contextes différents, pourront retenir. Il est notable de mentionner combien cette intuition est tout à fait cohérente avec le point de vue des chercheurs en Sciences de l'Education. Ainsi MEIRIEU (1991) considère que dans toute relation éducative institutionnalisée, il y a un "contrat", toutefois, à le lire, on constate bien que le contrat n'est pas nécessairement formalisé et que sa définition du "contrat" correspond parfaitement à la définition de la "convention" pour les économistes des conventions. SALAIS (1989) rappelait que le terme " convention », du latin " conventio » renvoyait à "convenir » et rendait compte d'un accord social qui n'est pas nécessairement explicite ${ }^{12}$.

\footnotetext{
${ }^{12}$ En comparant les pratiques routières en France, en Italie du Sud et au Liban, LEVY-TADJINE (2008a, pages 31-33), illustre comment la convention peut surpasser le texte explicitement admis (en l'occurrence le code de la route).
} 
Selon MEIRIEU (1991, p.105), “qu'on le veuille ou non, toute pédagogie est une pédagogie du contrat dans la mesure où elle gère tout un jeu d'attentes réciproques, souvent fort complexes, dans lequel interférent la position sociale des partenaires, les règles du jeu institutionnelles et leur interprétation locale ainsi que les contraintes spécifiques à la situation et à la discipline enseignée. Chacun attend donc quelque chose de l'autre, un type de comportement ou de réaction, un geste ou simplement un regard en réponse à chacune de ses demandes: chacun agit aussi en fonction de ce qu'il suppose que l'autre sait de ce qu'il attend." (Souligné par nous).

La parenté de cette définition avec celles de la convention est évidente. Il est temps d'esquisser une modélisation de la diversité des modèles de gouvernance universitaire. Celle-ci propose un croisement entre conventions de qualifications et conventions d'effort en démontrant que, pour un même jeu d'attentes des parties prenantes (la qualification), plusieurs réponses (effort) sont possibles. Pour simplifier et conformément aux propos précédents et au travail de GOMEZ, seules deux conventions d'effort et trois conventions de qualification seront croisées. Il en résulte six modèles types de gouvernance universitaire (Tableau 1). Les conventions de qualification sont a priori supportées par les diverses parties prenantes recensées (les étudiants; les futures étudiants ; les parents ; les enseignants ; le personnel administratif ; le gouvernement ; les collectivités locales ; les établissements concurrents ; les mass medias ; les organismes d'accréditation ; les fournisseurs d'équipement ; le monde professionnel ; etc) et il va sans dire qu'on pourrait alors superposer un troisième niveau de lecture. Selon les parties prenantes que les managers universitaires privilégieront, ils fixeront, en effet, l'une ou l'autre, des conventions de qualification. Ainsi, en reprenant la liste précitée de KOTLER \& FOX, on peut assimiler la convention marchande à une fixation sur les étudiants et les parents, la convention industrielle et du renom à une focalisation sur les enseignants, les mass médias et les organismes d'accréditation, et la convention domestique, à un accent sur les fondations, les anciens étudiants, les concurrents et le milieu professionnel. 


\begin{tabular}{|c|c|c|c|}
\hline & \multicolumn{3}{|c|}{ Conventions de qualification et parties prenantes les supportant } \\
\hline $\begin{array}{l}\text { Convention } \\
\text { d'effort }\end{array}$ & $\begin{array}{l}\text { Conventions marchandes } \\
\text { (couts ...) }\end{array}$ & $\begin{array}{l}\text { Conventions industrielles et du } \\
\text { renom } \\
\text { (standards techniques et opinion) }\end{array}$ & $\begin{array}{l}\text { Conventions domestiques } \\
\text { (réputation) }\end{array}$ \\
\hline $\begin{array}{l}\text { Université } \\
\text { centrée sur la } \\
\text { reconnaissance } \\
\text { individuelle }\end{array}$ & $\begin{array}{l}\text { A } \\
\text { Dans ce modèle qui valoriserait le } \\
\text { cout et la satisfaction immédiate } \\
\text { des étudiants avec une évaluation } \\
\text { individuelle des personnels, ceux-ci } \\
\text { seraient soumis à des évaluations } \\
\text { systématiques et à la pression de } \\
\text { ces dernières, l'institution assumant } \\
\text { un turn-over important à l'opposé } \\
\text { du modèle B. }\end{array}$ & $\begin{array}{c}\text { C } \\
\text { Les personnels se voient } \\
\text { assignés des objectifs } \\
\text { individuels à respecter (objectif } \\
\text { de publication ) à la différence } \\
\text { du modèle D dans lequel, les } \\
\text { objectifs sont fixés au niveau du } \\
\text { collectif. } \\
\text { Dans ce modèle centré sur } \\
\text { l'individu, l'atteinte des objectifs } \\
\text { suppose une gouvernance qui } \\
\text { valorise l'investissement et la } \\
\text { mise à disposition des ressources } \\
\text { et équipements satisfaisant les } \\
\text { standards techniques attendus. } \\
\end{array}$ & $\begin{array}{c}\text { E } \\
\text { La clef de la gouvernance } \\
\text { universitaire repose alors sur le } \\
\text { recrutement d'individus dotés d'une } \\
\text { grande notoriété et d'un fort pouvoir } \\
\text { d'attraction. } \\
\text { Il semble que ce soit en partie, le pari } \\
\text { de la jeune Faculté de gestion de } \\
\text { l'Université Antonine au Liban dont la } \\
\text { crédibilité repose pour l'heure, } \\
\text { presque exclusivement sur la } \\
\text { personnalité et sur le dynamisme de } \\
\text { son doyen, SEM Damianos Kattar, } \\
\text { ancien ministre des finances. }\end{array}$ \\
\hline $\begin{array}{l}\text { Université } \\
\text { centrée sur le } \\
\text { collectif }\end{array}$ & $\begin{array}{l}\text { B } \\
\text { Le manager est, dans ce modèle, un } \\
\text { véritable animateur d'équipe, } \\
\text { cherchant les synergies pour } \\
\text { répondre aux attentes des parties } \\
\text { prenantes. } \\
\text { Comme le modèle D, ce modèle } \\
\text { semble convenir à des } \\
\text { établissements faiblement dotés } \\
\text { budgétairement qui essaient de } \\
\text { compenser, de ce fait, } \\
\text { l'impossibilité à récompenser les } \\
\text { individus et à investir massivement } \\
\text { dans les équipements, par la } \\
\text { création d'une dynamique } \\
\text { collective. }\end{array}$ & $\begin{array}{c}\text { D } \\
\text { Le collectif reçoit un ensemble } \\
\text { d’objectifs à atteindre } \\
\text { collectivement (par exemple, un } \\
\text { certain nombre de } \\
\text { publications pour l'équipe; } \\
\text { l'obtention d'une accréditation à } \\
\text { un horizon fixé). Comme } \\
\text { mentionné plus haut, il nous } \\
\text { semble que la FGSc de l’USEK } \\
\text { se situe, ceteris paribus, dans ce } \\
\text { schéma }\end{array}$ & $\begin{array}{c}\text { F } \\
\text { La clef de la gouvernance } \\
\text { universitaire repose alors sur le } \\
\text { recrutement d'individus qui ne sont } \\
\text { pas nécessairement dotés d'une } \\
\text { grande notoriété et les dirigeants font } \\
\text { le pari de créer une notoriété } \\
\text { collective. } \\
\text { Dans certains cas, l'héritage historique } \\
\text { favorise cet état de fait. Au Liban, } \\
\text { l'AUB, l’USJ bénéficient } \\
\text { collectivement d'une grande } \\
\text { réputation en raison de leur histoire } \\
\text { sans pour autant que leurs } \\
\text { enseignants-chercheurs ne soient } \\
\text { individuelle ment réputés (excepté } \\
\text { dans le domaine hospitalo- } \\
\text { universitaire) }\end{array}$ \\
\hline
\end{tabular}

Tableau 1. Un modèle esquissant la diversité des conventions d'éducation

Quoi qu'il en soit, dés lors que, face à un même environnement, plusieurs réponses de gouvernance sont possibles même si pour simplifier la représentation, nous nous sommes limités à deux, les acteurs susceptibles de guider leur équipe vers l'un ou l'autre modèle, occupent une place centrale dans le processus de gouvernance universitaire. D'ailleurs, pour GOMEZ (1996), l'entrepreneur est celui qui sait convaincre d'autres acteurs pour créer avec eux une "convention d'effort ». Il nous faut donc partir à la recherche de l'acadépreneur. En première approche, ce dernier serait un acteur du système universitaire qui, par son action, innovante et fédératrice, permettrait à son établissement, de s'adapter ou même d'anticiper les attentes des parties prenantes.

La caractérisation de l'acadépreneuriat constitue, de ce fait, le deuxième étage d'un travail de refondation du concept de gouvernance universitaire. 


\section{A la recherche de l'acadépreneur}

L’émergence d'un néologisme n'est jamais anodine et traduit, bien souvent, un défi social. L'apparition du concept encore flou d'acadépreneuriat n'échappe pas à ce propos et rend compte d'un consensus sur la nécessité de valoriser certaines pratiques pour relever les nouveaux défis de la gouvernance universitaire. CLARK (1998) invitait ainsi, les universités, à devenir entrepreneuriales. CHAMPAGNE et CARRIER (2004) soulignaient, en ce sens, un "nouvel intérêt » pour "l'intrapreneuriat en contexte académique ». La problématique énoncée par ces auteurs, renvoyait explicitement à la gouvernance universitaire. Nos universités ont-elles besoin d'intrapreneurs, que ce soit pour créer des instituts ou chaires de recherche, développer de nouveaux programmes et des approches pédagogiques innovatrices?

En suivant LIU et DUBINSKY (2000), "University administrators are facing increasingly difficult time as public funds are contracting.... With these financial exigencies, universities must seek alternative means of generating revenues to support their mission. One such approach involves the use of institutional entrepreneurship."

La gouvernance universitaire supposerait alors d'encourager l'entrepreneuriat institutionnel $^{13}$. Le concept, issu de la sociologie économique, est cependant mal appréhendée comme le suggèrent LEVY-TADJINE, CHELLY, PATUREL (2006) et nécessite une clarification à laquelle nous entendons contribuer avant de pouvoir souligner les enjeux de l'acadépreneuriat qui, en suivant LIU et DUBINSKY, semble constituer une forme d'entrepreneuriat institutionnel appliquée au contexte académique (PATUREL, 2005 ; 2006 ; 2007 ; JAZIRI, 2007 ; JAZIRI et PATUREL 2008)

Pour Di MAGGIO (1988) et SUCKMAN (1995), l'entrepreneur institutionnel désigne l'acteur qui créée un nouveau contexte institutionnel ou manipule un contexte existant de manière à le redéfinir. En croisant cette perspective avec l'approche centrée sur l'émergence organisationnelle à la suite des travaux de GARTNER (1988), notamment développée en France par BRUYAT (1993), il est possible de distinguer là encore, plusieurs manifestations acadépreneuriales. BRUYAT (1993) dont les travaux font référence en France pour circonscrire le champ d'application des recherches en entrepreneuriat, propose de représenter le phénomène entrepreneurial dans les termes d'une dialogique complexe entre l'Individu (le Sujet) et la Création de Valeur Nouvelle qui constitue l'objet du processus entrepreneurial (BRUYAT, 1993, pp. 56-57), ce qui lui permet de construire une grille (récemment discutée et enrichie : PATUREL, 2005) sur laquelle il est possible de positionner les différentes définitions de l'entrepreneuriat (Figure 1).

\footnotetext{
${ }^{13}$ Comme le notait l'évaluateur de ce texte, l'adoption de cette perspective comporte aussi un versant « politique » non débattu dans ce texte mais dont les propos de LIU et DUBINSKY rendent bien compte. Le concept d'acadépreneur (ou d'entrepreneur institutionnel) est indissociable d'une vision très managériale de l'université qui postule d’une part que le déclin (relatif) des contributions publiques implique l'adhésion à une norme de rentabilité qui peut prendre des formes diverses et d'autre part que le « modèle étatique » conduit à une uniformisation préjudiciable. On peut d'ailleurs trouver une contestation radicale de cette démarche dans les travaux de LE GOFF (2003)
} 


\begin{tabular}{|c|c|c|c|}
\hline $\begin{array}{l}\text { Changement } \\
\text { important pour } \\
\text { l'Individu }\end{array}$ & & $\begin{array}{lrr}\text { Définition restrictive } & \text { de } \\
\text { l'entrepreneuriat limitée } & \text { à } \\
\text { l'entrepreneuriat innovant } & \end{array}$ & \begin{tabular}{|lrr} 
Définition restrictive & de \\
l'entrepreneuriat limitée & à \\
l'entrepreneuriat innovant & et \\
fortement créateur de valeur & \\
\end{tabular} \\
\hline $\begin{array}{l}\text { Changement moyen } \\
\text { pour l'Individu }\end{array}$ & & & $\begin{array}{lrr}\text { Définition restrictive } & \text { de } \\
\text { l'entrepreneuriat limitée } & \text { à } \\
\text { l'entrepreneuriat innovant } & \text { et } \\
\text { fortement créateur de valeur } & \\
\end{array}$ \\
\hline $\begin{array}{l}\text { Changement faible } \\
\text { pour l'Individu }\end{array}$ & $\begin{array}{l}\text { Zone ne caractérisant pas } \\
\text { l'entrepreneuriat }\end{array}$ & & \\
\hline & \begin{tabular}{lcc} 
Création & de & \multicolumn{2}{c}{ Valeur } \\
Nouvelle & faible & pour la \\
Société & &
\end{tabular} & $\begin{array}{l}\text { Création de Valeur Nouvelle } \\
\text { moyenne pour la Société }\end{array}$ & $\begin{array}{l}\text { Création de Valeur Nouvelle } \\
\text { élevée pour la Société }\end{array}$ \\
\hline
\end{tabular}

Figure 1: L'entrepreneuriat comme dialogique Individu/ Création de Valeur. BRUYAT (1993).

La transposition de cette modélisation au contexte acadépreneurial est évidente.

L'acadépreneur répond, en général, aux deux conditions requises selon BRUYAT pour caractériser l'entrepreneuriat : changement individuel important (I) et création de valeur élevée (CV), tout en respectant la définition originelle de l'etrepreneuriat institutionnel.

Deux exemples l'illustrent aisément.

M. R., universitaire reconnu dans son établissement, en acceptant un poste dans une nouvelle ville, pour y développer un institut supérieur d'enseignement et de recherche en gestion, a pris le risque d'altérer son image et a supporté un déménagement et un déplacement au sens de Shapero (I) tout en apportant à son nouvel environnement local, une offre de formation qui lui faisait défaut $(\mathrm{CV})^{14}$.

Dans un autre contexte, M. K., formateur dans une institution de l'Est de la France, en engageant un audit interne de sa structure a pris le risque d'être licencié lorsque ses résultats critiques (I) ont été publiés pour finalement, une fois la « digestion de l'audit » réalisée, contribuer à la ré-organisation de l'établissement (CV). (LEVY, 1998).

Conformément au travail fondateur de Di MAGGIO (1988) et SUCKMAN (1995), le premier a contribué à créer un nouveau contexte institutionnel local tandis que le second a manipulé un contexte existant de manière à le redéfinir.

Dès lors, JAZIRI et PATUREL (2008) définissent l'entrepreneuriat académique comme: "le phénomène par lequel un membre de l'université (étudiant, employé, chercheur, enseignant) crée ou reprend une activité nouvelle ou existante dépendante

14 A propos de cet acadépreneur, PATUREL (2006) affirmait qu' «un fonctionnaire de l'éducation nationale créant un institut de formation inexistant jusqu'ici dans la région où se trouve son université ou fondant un laboratoire de recherche utile pour le tissu économique local, se trouve sans ambiguïté dans une situation entrepreneuriale ». 
administrativement ou financièrement de l'université sans pour autant quitter cette dernière (acte d'intraprise), ou crée ou reprend une organisation juridiquement indépendante de l'université tout en abandonnant (éventuellement temporairement) cette dernière (acte d'extraprise). Pour pouvoir employer le terme d'acadépreneur, outre un changement plus ou moins intense qui doit exister pour le porteur de projet universitaire (seul ou en équipe) sur les plans statutaire, fonction, métier, géographique ou organisationnel, ce dernier doit capter de la valeur nouvelle (en cas de création exnihilo) ou existante (en cas de reprise) au sein de l'entité qu'il impulse. »

Seule la promotion de ce type d'acteurs peut permettre l'évolution des conventions d'effort et leur adaptation aux nouvelles conventions de qualification du marché universitaire. Les nouvelles lois sur l'autonomie des universités (en France notamment) comme les rapports sur lesquels elles s'appuient, attestent de la pertinence éprouvée de cette conviction. Il n'en demeure pas moins que les managers universitaires ont encore le choix de privilégier un acadépreneuriat collectif ou un acadépreneuriat individuel et qu'au sein des systèmes universitaires, certains pourraient s'opposer aux initiatives acadépreneuriales. Comme toutes les organisations, les universités sont aussi des espaces susceptibles de générer des mécanismes de résistance au changement qui mériteraient un examen spécifique.

\section{Conclusion}

Prenant acte des nouveaux défis que pose la démultiplication des parties prenantes universitaires et des missions assignées à l'université, cette contribution a suggérer conceptuellement comment la mobilisation de la théorie des conventions et du concept d'acadépreneuriat pouvaient contribuer à renouveler le débat sur la gouvernance universitaire. Le travail de discussion et de modélisation engagé demeure exploratoire et gagnerait à être poursuivi, notamment afin d'identifier plus précisément, les contours des différentes "conventions d'éducation » et leurs conditions d'émergence. Nul doute que l'environnement explique celle-ci et on pourrait légitimement s'interroger sur le fait de savoir si un mode de gouvernance tendrait à s’imposer - même tendanciellement - dans un contexte de mondialisation de l'enseignement supérieur. AMABLE (2007) considère ainsi que le " modèle anglo-saxon » serait en train de supplanter le "modèle européen ». La reprise de sa discussion à la lumière de nos modélisations des «conventions d'éducation » constituerait un programme de recherches à part entière ${ }^{15}$. Pour autant, le résultat majeur de ce travail tient dans l'affirmation d'une pluralité des modèles de gouvernance. Sur le plan managérial et politique, cette conclusion critique les tendances totalitaires à l'uniformisation et l'utopie de référentiels uniques et universels susceptibles de garantir la qualité des systèmes universitaires.

Le soubassement philosophique de cette critique relève d'une réflexion sur « le juste ». La gouvernance renvoie, en effet, à l'évaluation des actions et à leur justification. Certains s'émeuvent, en effet, de l'application du concept de gouvernance à d'autres domaines de la sphère publique que la seule entreprise. Ainsi, pour CROWLEY (2003),

\footnotetext{
${ }^{15}$ Pour donner crédit à sa thèse, il conviendrait, en effet, de caractériser les « conventions » anglo-saxonnes et de les distinguer de leurs homologues « européennes ».
} 
parler de gouvernance dans la sphère publique, conduit à vider l'Etat et le politique de leurs responsabilités et de leur souveraineté en réduisant ceux-ci à de simples acteurs économiques dans une perspective ultra-libérale.

"Dès lors que tout système organisé (un État, une entreprise, une université, une association, une ville...) peut être conçu comme doté d'une "gouvernance », et dès lors que la gouvernance a vocation à constituer une grille d'analyse privilégiée pour la compréhension des logiques de pouvoir et de production d'effets dans un tel système, l'État se voit nécessairement retirer tout statut exorbitant. Il est vrai que, en toute rigueur, cette désacralisation analytique est indépendante du statut normatif de l'État. (...) Le monde de la gouvernance est ainsi un monde d'où la souveraineté est absente.». En mobilisant l'axiomatique de la justice locale ${ }^{16}$ (BOLTANSKI et THEVENOT, 1987) pour aborder la gouvernance universitaire, nous pensons avoir contribué à lever cette objection d'une non-applicabilité du concept de gouvernance au système éducatif et surtout avoir démontré combien les managers des institutions d'enseignement supérieur conservaient une immense responsabilité de par le choix des conventions d'éducation sur lequel ils influent. L'enjeu des nouveaux fondements proposés au concept de gouvernance n'est donc pas anodin. De nombreuses pistes d'approfondissement subsistent pour autant. Ce travail a interrogé le concept de gouvernance universitaire en partant de quelques caractéristiques de l'environnement moderne. Sans pour autant questionner l'influence des nouvelles technologies de l'information. Un tel projet constitue un programme de recherche à part entière. Les universités sont, en effet, contraintes d'intégrer dans leur mode de gouvernance, des outils et des stratégies pour lutter la tentation de plagiat chez les étudiants tout en prenant en compte, dans leurs programmes pédagogiques, le fait que via internet, les étudiants et les autres parties prenantes ont accès à de nombreuses informations. Les nouvelles technologies modifient de fait, le statut de l'étudiant et questionnent le pédagogue. Inutile de rappeler, par exemple, comment l'accès aux informations du web rend moins attractives pour l'étudiant, la visite en bibliothèque. L'enseignant universitaire du 21eme siècle peut-il s'en satisfaire ? Telle serait une des voies d'approfondissement de notre travail sur la gouvernance universitaire. L'autre concerne l'acadépreneuriat en envisageant notamment, dans le prolongement de ce travail, la distinction entre ses manifestations individuelles et collectives. On pourrait alors mobiliser l'axiomatique proposée par LEVY-TADJINE (2008b) pour appréhender la singularité de l'entrepreneuriat collectif. Ceci permettrait un approfondissement du modèle des conventions d'éducation qui repose sur la dualité des conventions d'effort.

\footnotetext{
${ }^{16}$ Comme le note ELSTER (1991), a l’opposé de la « théorie de la justice globale » qui animent les débats de la philosophie politique moderne (RAWLS, 1971; SANDEL, 1982), les approches de la « justice locale », mettent l'accent sur l'existence d'une pluralité de principes de justice comme le font notamment les auteurs de la Théorie des Conventions et, par exemple, BOLTANSKI et THEVENOT (1987, 1991), mobilisés dans ce travail. Le lecteur intéresse par les enjeux de ce débat philosophique peut se reporter aux chapitres 2 et 3 de LEVY-TADJINE (2004) dans lesquels l'opposition entre les deux conceptions du juste est présentée.
} 


\section{Références}

AMABLE B. (2007), La fin du modèle européen ?, Revue d'Economie Politique, juilletaoût 2007.

BELET D. (1991), Changer les comportements managériaux par la formation : Pour une andragogie du management, Cahiers de recherche du CREGE, No I.9103, Université Bordeaux I.

BEN-HAMOUDA S., PATUREL R. (2009), Les institutions publiques d'enseignement supérieur (IPES), leurs parties prenantes et leur marché: vers un nécessaire rapprochement, contribution au colloque La Gouvernance Universitaire, USEK.

BOUCHIKI H. (2004), Quels fondements pour une identité professionnelle de l'Entrepreneuriat dans les Sciences de Gestion ?, Conférence d'ouverture du $3^{\text {ème }}$ congrès de l’Académie de l’Entrepreneuriat, Lyon, 31 Mars-1 ${ }^{\mathrm{er}}$ Avril 2004.

BRUYAT C. (1993), Création d'entreprise: contributions épistémologiques et modélisation., Thèse de doctorat ès sciences de gestion, Université Pierre-MendezFrance, Grenoble 2.

CHAMPAGNE D., CARRIER C. (2004), Les études sur l'intrapreneuriat: objets d'intérêt et voies de recherche, $7^{\text {ème }}$ CIFPME-27, 28 et 29 Octobre, Montpellier.

CLARK B.R. (1983), The higher education system : academic organization in crossnational perspective, The University of California Press, Berkeley, California.

CLARK B.R. (1987), The Academic life; Small Worlds, Different worlds, Carnegie Foundation, Princeton, cite in LOSEGO (2004).

CLARK, B.R. (1998), Creating Entrepreneurial Universities. London: Pergamon Press,1998.

COULON A. (2004), L'Ecole de Chicago, PUF.

CROWLEY J. (2003), Usages de la gouvernance et de la gouvernementalité, Critiques Economiques, 21.

DEROUET J.L. (1992): “Ecole et Justice. De l'égalité des chances aux compromis locaux.” Metaillé, Paris.

Di MAGGIO P. (1988), Interest and agency in institutional theory, in L.G. ZUCKER (Ed), Institutional patterns and organization culture and environment ; 3-21 ; Ballinger Publishing Co; Cambridge, Massachussets. 
DRUCKER P. (1957), La pratique de la direction des entreprises, Editions d'Organisations, Paris.

ELSTER J. (1991), Local Justice, Cambridge University Press.

ESPERET E. (2001), Nouvelle définition des taches des enseignants et des enseignantschercheurs dans l'enseignement supérieur français, Ministère de l'Education Nationale.

EYMARD-DUVERNAY F. (1989), Conventions de qualité et formes de coordination, Revue Economique, Mars.

FREIDSON E. (1984), La profession médicale, Payot, Paris.

GARTNER W.B. (1988), "Who is an entrepreneur?” is the wrong question, American Journal of Small Business, 12 (4), Spring, p. 11-31

GOMEZ P.Y., (1994), Qualité et Théorie des organisations, Economica, Paris.

GOMEZ P.Y., (1996), Le gouvernement d'entreprise, Les éditions d’Organisation, Paris.

JAZIRI, R. (2007). Peut-on parler de l'intrapreneuriat dans une organisation complexe comme l'université : vers l'acadépreneuriat. Actes du $4{ }^{\text {ème }}$ Colloque International de Fiance (IFC). 15-16-17 mars 2007. Hammamet Sud. Tunisie.

JAZIRI, R. PATUREL, R. (2008), Peut-on être entrepreneur quand on est membre de l'université : vers une délimitation du concept de l'acadépreneuriat. Actes de la $1^{\text {ère }}$ conférence internationale sur « la culture entrepreneuriale et création d'entreprises : les pratiques et les méthodes pédagogiques de la formation en entrepreneuriat à l’université ». 27-30 mars 2008. Monastir.

KOTLER P., FOX K. (1995), Strategic marketing for Educational Institutions, Edition Prentice-Hall, Englewood Cliffs, 2ème Edition, New Jersey, 464 p.

LE GOFF J.Y. (2003), La barbarie douce, La découverte, Paris.

LEVET J.L. (1999), Sept leçons d'économie a l'usage du citoyen, Editions du Seuil, Paris.

LEVY T. (1998), Démarche Qualité, Conventions et Audit de type socio-économique dans les institutions éducatives du Supérieur. (Récit et Analyse d'une intervention menée à l’IUT d’Hellespont), Revue Savoirs-Education-Formation, Nº1, 1998, pp. 69-96

LEVY T. (2002), The Theory of conventions and a new theory of the firm, in E. FULLBROOK (Ed), Intersubjectivity in Economics, agents and structures, Routledge. 
LEVY-TADJINE T. (2004), L'entrepreneuriat immigré et son accompagnement en France, Thèse de Doctorat en Sciences de Gestion, Université du SUD-Toulon-Var, Prix FNEGE 2004 de la meilleure thèse en entrepreneuriat; Nominée parmi les cinq meilleures thèses d'Economie-Gestion par l'ANDESE (Association Nationale des Docteurs en Sciences Economiques et de Gestion) ; téléchargeable en ligne : http://www.pseau.org/outils/biblio/resume.php?docu_document_id=233 http:// asso.nordnet.fr/adreg/levy_tot_sans_annexe.pdf

LEVY-TADJINE T. , CHELLY A., PATUREL R. (2006), Pour déconstruire le concept d'entrepreneuriat institutionnel et ses utilisations abusives en Management Stratégique, Communication présentée au CIFEPME 2006, Fribourg, 25-27 octobre.

web.hec.ca/airepme/images/File/2006/021_Pourdesconstruire.pdf

LEVY-TADJINE T. (2008a), Témoin au Liban avec le Hezbollah, L’Harmattan, Paris.

LEVY-TADJINE T. (2008b), Action collective et entrepreneuriat: Proposition d'un cadre conceptuel pour appréhender l'entrepreneuriat en équipe, Revue Organisation et Territoires, Vol 17, №2 (Eté), pp 57-62.

LOSEGO P. (2004), Le travail invisible a l'université : le cas des antennes universitaires, Sociologie du Travail, 46, 2, 187-204.

MEIRIEU P. (1991), Le choix d'éduquer, ESF éditeur, Paris.

PATUREL R. (2005), Pistes de réflexion en vue de l'élaboration d'une grille de positionnement des pratiques de l'entrepreneuriat, $4^{\text {ème }}$ Congrès de l'Académie de l'Entrepreneuriat sur «L'accompagnement en situation entrepreneuriale : pertinence et cohérence », 24-25 novembre, 2005, Actes sur le site de l’AE, 23 pages.

PATUREL R. (2006), Délimitation du champ de l'entrepreneuriat par ses caractéristiques, pratiques et paradigmes, $1^{\mathrm{er}}$ colloque International du Réseau Méditerranéen des Ecoles de Management, «Le management dans l'Espace Méditerranéen : Modèles et Pratiques », 9 et 10 juin 2006, Beyrouth, actes.

PATUREL R. (2007), Grandeurs et servitudes de l'entrepreneuriat, Editorial du $\mathrm{n}^{\circ}$ spécial de la Revue Internationale de Psychosociologie consacré aux Représentations entrepreneuriales, Direction Robert PATUREL, Christophe SCHMITT et Christian BOURION, Volume XIII, $n^{\circ}$ 31, Hiver 2007, pages 27-43.

RAWLS J. (V.O. :1971 ; V.F. : 1987), Théorie de la Justice, Seuil, Paris.

SALAIS R. (1989), Conventions et marché du travail, Revue Economique, Vol. 40, N², Mars 1989.

SANDEL M. (1982), Liberalism and the limits of justice, Cambridge university Press, Cambridge. Traduction française 1999 : Le Libéralisme et les limites de la justice, Seuil, Paris. cité par -WIEVIORKA M. (2001), La différence, Balland. 
SAVALL H., ZARDET V., (1991), Maîtriser les coûts et les performances cachés, Economica.

STROOBANTS M. (1999), Sociologie du Travail, Nathan, Paris.

SUCHMAN M.C. (1995), Managing legitimacy: Strategic and institutional approaches, Academy of Management Review; 20 (3); 571-610. 